\title{
El concepto de exactitud científica en las especialidades clínicas
}

\author{
M. ORTEGA CALVO
}

Centro de Salud Esperanza Macarena. Sevilla

\section{RESUMEN}

¿Tendrán las especialidades clínicas un estatuto epistemológico de ciencia en el futuro? Sí, lo tendrán mediante una transformación de tipo continuo. El concepto clínico de exactitud es muy posible que sea diferente gracias a las aportaciones lógico-formales de las matemáticas, al desarrollo de la física cuántica y a las aplicaciones de la teoría de la relatividad. La medicina basada en la evidencia está ayudando al tránsito hacia la "exactitud científica" de las especialidades clínicas sobre todo en el campo de la teoría de la decisión.

PALABRAS CLAVE: Ciencia. Exactitud. Medicina basada en la evidencia. Análisis bayesiano. Especialidades clínicas. Decisión.
UPON SCIENTIFIC ACCURACY SCHEME AT CLINICAL SPECIALTIES

\section{ABSTRACT}

Will be medical specialties like sciences in the future? Yes, progressively they will. Accuracy in clinical specialties will be dissimilar in the future because formal-logic mathematics, quantum physics advances and relativity theory utilities. Evidence based medicine is now helping to clinical specialties on scientific accuracy by the way of decision theory.

KEY WORDS: Science. Accuracy. Evidence based medicine. Bayesian analysis. Medical specialties. Decision.

Ortega Calvo M. Concepto de exactitud científica en las especialidades clínicas. An Med Interna (Madrid) 2006; 23: 552-554.

\section{INTRODUCCIÓN}

Los profesionales sanitarios que tratamos a pacientes, estamos acostumbrados a comportamientos de tipo desorganizado en algunos de ellos $(1,2)$. Estas experiencias nos hacen dudar cuando alguien nos intenta convencer de la naturaleza científica de nuestro ejercicio diario. La cuestión real es saber cómo podemos cuantificar el cientifismo de la medicina clínica y cómo podemos precisar la exactitud de respuesta ante los mismos estímulos tanto en la esfera diagnóstica como en el campo de la terapéutica. En otras palabras, ¿cuál es el concepto de exactitud científica que posee aún el médico clínico en la actualidad.

\section{ACLARACIONES}

La idea de ciencia en el mundo occidental recoge cuatro significados diferentes que han ido desarrollándose históricamente $(3,4)$. La primera acepción es la de "saber hacer" (la ciencia del "navegante" o la del "tonelero"). El segundo sentido tiene un origen aristotélico y está fraguado sobre el patrón de la geometría euclidiana. Se puede considerar un equivalente de la voz griega "episteme" y se presenta como "un sistema de proposiciones derivadas de unos principios". La tercera acepción supera a las dos anteriores y contempla exclusivamente a las "ciencias positivas" surgidas en la Edad Moderna, tanto "empíricas" (física, química, biología) $(5,6)$ como "lógico-formales" (las matemáticas) sobre todo a partir del estímulo cualitativo que supusieron para estas últimas los autores lógicos del siglo XIX y del XX (7). El método de trabajo filosófico en este caso es el método científico (2). El cuarto significado es una extensión del anterior. Trata sobre prácticas y realidades que ya no son empírico-naturales de forma estricta ni tampoco abstracto-formales, sino genuinamente humanas y sociales (lingüística, economía, historia) dando origen a un vocablo "nuevo", las ciencias humanas, ciencias sociales o ciencias culturales $(3,4)$.

Trabajo aceptado: 22 de septiembre de 2006 
Las especialidades clínicas (medicina de familia, medicina interna, pediatría, ginecología, cirugía general y digestiva...) caen de lleno dentro de las dos últimas acepciones de ciencia, la empírico-formal y la humana. En la primera, porque su base de conocimiento está radicada en la biología y en la química que son ciencias empíricas (8). En la segunda porque la medicina clínica aunque no es todavía una ciencia humana, sobre todo en sus fases diagnóstica y terapéutica, lo será más tarde o más temprano $(3,9)$.

\section{ANTECEDENTES HISTÓRICOS}

Los antecedentes científicos de la medicina clínica están sin duda en el avance que tuvieron las disciplinas básicas desde el Renacimiento. La anatomía con las aportaciones de Andrea Vesalio (1514-1564), la fisiología con las de William Harvey (1578-1657) y la terapéutica con las de Paracelso (1493-1541), por citar algunas. En el terreno estrictamente clínico merecen recordarse a Thomas Sydenham (1624-1689), a Hermann Boerhaave (1668-1738) y a Georg Ernst Stahl (1659-1734) por su labor sistematizadora (10). En cierta forma fueron los introductores del concepto clasificatorio en la embrionaria "ciencia" clínica. En el siglo XIX apareció la figura singular de Pierre Charles Alexandre Louis (1787-1872), a quien por su labor crítica sobre las sangrías (un tratamiento muy utilizado en su tiempo para toda clase de problemas) se le imputa un papel profético de la medicina basada en la evidencia (11).

\section{CONCEPTO DE EXACTITUD Y TEORÍA DE LA MEDIDA}

¿Serán las especialidades clínicas más exactas en el futuro? Es fácil aventurar que sí. Pero creemos que el camino será largo (quizás de uno o dos siglos) y sobre todo que será un recorrido de tipo continuo ("paso a paso"). Hemos de plantearnos ahora otra pregunta. ¿El concepto de exactitud $(4,5)$ al que queremos que lleguen las especialidades clínicas en el futuro será el mismo que tenemos ahora? Posiblemente no. Quizás sea un concepto diferente.

El concepto de exactitud científica que manejamos en la actualidad los clínicos es un concepto de naturaleza "euclidiana" o si nos permiten "aritmética". El concepto de exactitud que manejan las ciencias lógico-formales (las matemáticas) y la física en la actualidad es un concepto de diferente naturaleza y menos rígido. Contribuyeron a ello una serie de investigadores de finales del siglo XIX y del siglo XX (7). Merecen citarse entre otros a Gottlob Frege, Georg Cantor, Bertrand Russell, Von Neuman y Kurt Gödel. Ellos libraron a las matemáticas de la rigidez euclidiana clásica para transformarlas en transacciones lógico-formales en donde el valor aritmético del número no es decisivo. Profundizar en esta idea se sale claramente de los límites de este trabajo, pero quien quiera hacerlo puede consultar las obras de Mosterín $(7,12,13)$. Se postula también desde hace años la existencia de una "aleatoriedad" intrínseca a la aritmética, basándose en elementos de programación informática y de termodinámica clásica $(14,15)$.

Si nos asomamos a la física, la revolución quizás haya sido más ostensible. En un corto periodo de tiempo, el que fué desde el año 1900 hasta el año 1905 ocurrieron dos hechos trascendentales para la historia de la ciencia que trastocaron la idea de exactitud en la medida. El primero fue la aparición de la física cuántica ideada por Max Planck y el segundo la aparición de la teoría especial de la relatividad de Albert Einstein (16). Tratar en este lugar los conceptos de "quanta", de función de onda o de la curvatura del espacio-tiempo también excede claramente de los límites de este artículo (16). Ambas aportaciones fueron fundamentales para la erosión del concepto euclidiano de exactitud en la teoría de la medida física (5).

\section{ESTADÍSTICA BAYESIANA Y MEDICINA BASADA EN LA} EVIDENCIA (MBE)

Thomas Bayes no aquilató bien antes de fallecer la influencia que dos siglos más tarde iba a tener su ensayo sobre la probabilidad condicionada. Tratar en este lugar las aportaciones de Blas Pascal, de Ferdinand Laplace o de Quetelet al concepto de probabilidad sanitaria excede también los límites de este artículo (11). La medicina clínica de finales del siglo XX hubiera sido otra sin el teorema de Bayes. El desarrollo de la epidemiología moderna de los factores de riesgo o de la teoría del ensayo clínico habría sido diferente.

Había tanta información biomédica a principios de la década de los noventa que los internistas y los epidemiólogos de la Universidad MacMaster de Canadá tuvieron que avisar a sus futuros residentes de que la formación que iban a recibir allí iba a ser "escéptica y crítica" $(17,18)$ frente al aluvión de datos y experimentos recogidos en la bibliografía. Mediante las técnicas de la epidemiología clínica, se iban a buscar los hallazgos más fuertes en las diferentes especialidades y se iban a crear protocolos de decisión en base a ellos. Acababa de nacer la MBE que tan buenos resultados ha estado provocando en la medicina clínica en los últimos años a pesar de las críticas filosóficas que se le puedan imputar (17). La MBE ha de tener en cuenta al paciente, sus inclinaciones y sus preferencias $(18,19)$, dándole un matiz claro de ciencia humana a la medicina clínica.

\section{UNA TEORÍA CRÍTICA DE LA MEDICINA CLÍNICA}

Soriguer Escofet refiere (20) que: "La aparente contradicción entre la reivindicación de una teoría científica para la práctica clínica y el reconocimiento de la imposibilidad de una práctica exclusivamente científica es lo que justifica y define una teoría crítica de la medicina clínica". Pensamos que cuando existan los instrumentos que permitan sortear esa contradicción, la medicina clínica será científica. Es curioso que las especialidades clínicas gozan en la actualidad de un estatus no científico y que están esperando su revolución en el sentido kuhniano de la palabra para transformarse en ciencia normal (21). Pero esa revolución será continua, no será a nuestro entender una revolución discreta. Con ello no nos atribuimos ninguna originalidad. William Whewell (1794-1866) creía hace muchos años en la progresión permanente de las ciencias mediante la analogía del río y sus afluentes $(16,22)$.

\section{EL FUTURO}

Nosotros creemos que las especialidades clínicas alcanzarán el estatuto epistemológico de ciencia en un futuro más o menos cercano (Tabla I). Se conseguirá gracias a la depuración filosófica de su teoría crítica (20), gracias al cambio en la naturaleza 
del concepto clínico de exactitud (transitando desde el euclidiano al lógico-formal y del newtoniano al cuántico-relativista), gracias a la propia inercia en el desarrollo de las diversas especialidades $(10,16)$, gracias a la implantación del modelo de decisión probabilística (11) y finalmente gracias a la introducción de la epidemiología clínica dentro de los programas de formación de pre y postgraduados (23). Parece, no obstante, que falta camino por recorrer. Reid y colaboradores expusieron a finales de los noventa que menos del cinco por ciento de los médicos tomaban decisiones con arreglo a criterios cuantitativos propios de la epidemiología clínica (24). Falta todavía algo de tiempo para la transformación definitiva.

\section{Bibliografía}

1. Goldberger AL, Rigney DL, West BJ. Caos y fractales en la fisiología humana. Investigación y Ciencia (edición española) 1990; 163: 30-38.

2. Trilla A. El caos y la predicción médica. Med Clin (Barc) 1998; 110: 619-620.

3. Moradiellos E. Las caras de Clío. Una introducción a la Historia. Madrid: Siglo Veintiuno de España Editores. S.A. 2001. p. 21-48.

4. Bueno G. Teoría del cierre categorial. Introducción General. Siete enfoques en el estudio de la Ciencia. Volumen I. Oviedo: Pentalfa; 1992.

5. Omnés R. Filosofía de la ciencia contemporánea. Barcelona: Idea Books. S.A. 2000

6. Strathern P. El sueño de Mendelèiev. De la alquimia a la química. Madrid: Siglo Veintiuno de España Editores. S.A. 2000.

7. Mosterín J. Los lógicos. Espasa Forum. Madrid: Espasa Calpe S.A. 2000.

8. Sierra Bravo R. Tesis doctorales y trabajos de investigación científica. Thomson. Madrid: Paraninfo S.A. 2002. $5^{\mathrm{a}}$ ed.

9. Starfield B. Atención Primaria. Equilibrio entre necesidades de salud, servicios y tecnología. Masson. S.A. 2001.

10. Laín Entralgo P. Historia de la Medicina. Barcelona: Salvat Editores S.A. 1978.

11. Almenara Barrios J, Silva-Ayçaguer LC, Benavides Rodríguez A, García Ortega C, González Caballero JL. Historia de la bioestadística: la génesis, la normalidad y la crisis. Cádiz: Quórum Editores; 2003. p. 5254.

12. Mosterín J. Conceptos y Teorías de la Ciencia. Colección Ensayo. Madrid: Alianza Editorial S.A. 2000.

13. Mosterín J. Ciencia viva. Reflexiones sobre la aventura intelectual de

\section{TABLA I}

RAZONES POR LAS QUE LAS ESPECIALIDADES CLÍNICAS SERÁN CIENTÍFICAS EN EL FUTURO

—Depuracion filosófica de su teoría crítica

- Transformación del concepto clínico de exactitud

- Inercia propia en su desarrollo histórico

- Implantación del modelo de decisión probabilistico

-Introducción definitiva de la Epidemiología Clínica nuestro tiempo. Espasa Forum. Madrid: Espasa Calpe S.A. 2001.

14. Chaitin GJ. Aritmética y azar. Investigación y Ciencia (edición española) 1988; 144: 44-50.

15. Chaitin GJ. Ordenadores, paradojas y fundamentos de las matemáticas. Investigación y Ciencia (edición española) 2003; 322: 28-35.

16. Losee J. Introducción histórica a la filosofía de la ciencia. Alianza Universidad. $1^{\mathrm{a}}$ ed. $7^{\mathrm{a}}$ reimp. 2000.

17. Ortega Calvo M, Cayuela Domínguez A. Medicina Basada en la Evidencia: Una crítica filosófica sobre su aplicación en Atención Primaria. Revista Española de Salud Pública 2002; 76: 115-120.

18. Guyatt G. Preface. In: Guyatt G, Rennie D, editorss. User's Guide to the Medical Literature. Essentials of Evidence Based Medicine Clinical Practice. AMA Press. USA; 2002. p. XV-XX.

19. Hanning G, George J. Teaching Evidence-based Medicine in a small rural Family Practice Office. Fam Med 2003; 35: 241-242.

20. Soriguer Escofet F. ¿Es la clínica una ciencia? Madid: Ediciones Díaz de Santos. S.A. 1993. p. 35-49.

21. Mosterín J, Torretti R. Diccionario de Lógica y Filosofía de la Ciencia. Voces "ciencia normal" y "cientifismo". Madrid: Alianza Editorial. S. A. 2002. p. 89-90.

22. Whewell W. History of the Inductive Sciences. New York: Johnson Reprint Corp; 1847.

23. Fletcher RH, Fletcher SW, Wagner EH. Epidemiología Clínica. Aspectos Fundamentales. Masson. William \& Wilkins. España. S.A. $2^{\text {a }}$ ed. 1998.

24. Reid MC, Lane DA, Feinstein AR. Academic calculations versus clinical judgments: Practicing physicians' Use of quantitative measures of test accuracy. Am J Med 1998; 104: 374-380. 